\title{
Mobile phone data for informing public health actions across the COVID-19 pandemic lifecycle
}

\author{
Nuria Oliver ${ }^{1,2}$, Bruno Lepri $^{2,3}$, Harald Sterly $^{4}$, Renaud Lambiotte $^{5,6}$, Sébastien Delataille $^{7}$, Marco De Nadai $^{3}$, \\ Emmanuel Letouzé $^{2,8}$, Albert Ali Salah ${ }^{2,9}$, Richard Benjamins ${ }^{10,11}$, Ciro Cattuto ${ }^{12,13}$, Vittoria Colizza ${ }^{14}$, Nicolas de \\ Cordes $^{15}$, Samuel P. Fraiberger ${ }^{16}$, Till Koebe ${ }^{2,17}$, Sune Lehmann ${ }^{18}$, Juan Murillo ${ }^{19}$, Alex Pentland ${ }^{20}$, Phuong $\mathbf{N}$ \\ Pham $^{2,21}$, Frédéric Pivetta ${ }^{22}$, Jari Saramäki ${ }^{23}$, Samuel V. Scarpino ${ }^{24}$, Michele Tizzoni ${ }^{12}$, Stefaan Verhulst ${ }^{25}$, \\ Patrick Vinck ${ }^{2,21^{*}}$
}

\begin{abstract}
${ }^{1}$ ELLIS, the European Laboratory for Learning and Intelligent Systems ${ }^{2}$ Data-Pop Alliance (USA, Colombia, Mexico, Senegal and Spain) ${ }^{3}$ Fondazione Bruno Kessler, Italy 4 University of Vienna, Austria ${ }^{5}$ University of Oxford, UK ${ }^{6}$ Turing Institute, UK ${ }^{7}$ Rosa, Belgium ${ }^{8}$ OPAL Project ${ }^{9}$ Utrecht University, Netherlands ${ }^{10}$ Telefonica, Spain ${ }^{11}$ OdiselA, Spain ${ }^{12}$ University of Turin, Turin, Italy ${ }^{13}$ ISI Foundation, Italy ${ }^{14}$ INSERM, Sorbonne Université, Pierre Louis Institute of Epidemiology and Public Health, Paris, France ${ }^{15}$ Orange Group, France ${ }^{16}$ World Bank, Washington DC, USA ${ }^{17}$ Freie University, Berlin, Germany ${ }^{18}$ Technical University of Denmark, Copenhagen, Denmark ${ }^{19}$ Banco Bilbao Vizcaya Argentaria, Madrid, Spain ${ }^{20}$ Massachusetts Institute of Technology, Cambridge, USA ${ }^{21}$ Harvard University, Cambridge, USA ${ }^{22}$ Dalberg Data Insights, Belgium ${ }^{23}$ Aalto University, Finland ${ }^{24}$ Northeastern University, Boston, MA, USA ${ }^{25}$ The GovLab, New York University, USA

*Corresponding author. Email:_pvinck@hsph.harvard.edu
\end{abstract}

The coronavirus 2019-2020 pandemic (COVID-19) poses unprecedented challenges for governments and societies around the world (1). Non-pharmaceutical interventions (NPIs) have proven to be critical for delaying and containing the COVID-19 pandemic (2-6). This includes testing and tracing, bans on large gatherings, non-essential business and school and university closures, international and domestic mobility restrictions and physical isolation, and total lockdowns of regions and countries. Decision-making and evaluation or such interventions during all stages of the pandemic lifecycle require specific, reliable and timely data not only about infections, but also about human behavior, especially mobility and physical co-presence. We argue that mobile phone data, when used properly and carefully, represents a critical arsenal of tools for supporting public health actions across early, middle, and late-stage phases of the COVID-19 pandemic.

Seminal work on human mobility has shown that aggregate and (pseudo-)anonymized mobile phone data can assist the modeling of the geographical spread of epidemics (7-11). Thus, researchers and governments have started to collaborate with private companies, most notably mobile network operators and location intelligence companies, to estimate the effectiveness of control measures in a number of countries, including Austria, Belgium, Chile, China, Germany, France, Italy, Spain, UK and the US (12-21).

There is, however, little coordination or information exchange between these national or even regional initiatives (22). Although ad hoc mechanisms leveraging mobile phone data can be effectively (but not easily) developed at the local or national level, regional or even global collaborations seem to be much more difficult given the number of actors, the range of interests and priorities, the variety of legislations concerned, and the need to protect civil liberties. The global scale and spread of the COVID-19 pandemic highlight the need for a more harmonized or coordinated approach.

In the following sections, we outline the ways in which different types of mobile phone data can help to better target and design measures to contain and slow the spread of the COVID-19 pandemic. We identify the key reasons why this is not happening on a much broader scale, and we give recommendations on how to make mobile phone data work against the virus.

\section{How can mobile phone data help to tackle the COVID-19 pandemic?}

Passively generated mobile phone data have emerged as a potentially valuable data source to infer human mobility and social interactions. Call Details Records (CDRs) are arguably the most researched type of mobile data in this context. CDRs are collected by mobile operators for billing purposes. Each record contains information about the time and the cell tower that the phone was connected to when the interaction took place. CDRs are event-driven records: in other words, the record only exists if the phone is actively in use. Additional information includes 'sightings data' obtained when a phone is seen on a network. There are, however, other types of mobile phone data used to study human mobility behaviors and interactions. X Data Records (XDRs) or network probes, can be thought as metadata about the phone's data channel, capturing background actions of apps and the network. Routine information including highly accurate location data are also collected through mobile phone applications (Apps) at a large scale by location intelligence companies (23) or by ad hoc applications $(24,25)$. Additionally, proximity between mobile phone users can be detected via Bluetooth functionality on smartphones. Each of these data types requires different processing frameworks and raise complex ethical 
and political concerns that are discussed in this paper.

First, we explore the value and contribution of mobilephone data in analytical efforts to control the COVID-19 pandemic. Government and public health authorities broadly raise questions in at least four critical areas of inquiries for which the use of mobile phone data are relevant. First, situational awareness questions seek to develop an understanding of the dynamic environment of the pandemic. Mobile phone data can provide access to previously unavailable population estimates and mobility information to enable stakeholders across sectors better understand COVID-19 trends and geographic distribution. Second, cause-and-effect questions seek to help identify the key mechanisms and consequences of implementing different measures to contain the spread of COVID-19. They aim to establish which variables make a difference for a problem and whether further issues might be caused. Third, predictive analysis seeks to identify the likelihood of future outcomes and could, for example, leverage real-time population counts and mobility data to enable new predictive capabilities and allow stakeholders to assess future risks, needs, and opportunities. Finally, fourth, impact assessments aim to determine which, whether, and how various interventions affect the spread of COVID-19 and require data to identify the obstacles hampering the achievement of certain objectives or the success of particular interventions. Table 1 provides specific examples of questions by areas of enquiry.

The relevance and specific questions raised as part of these areas of inquiry differs at various stages of the outbreak, but mobile phone data provide value throughout the epidemiological cycle, shown in Fig. 1.

In the early recognition and initiation phase of the pandemic, responders focus on situational analysis and the fast detection of infected cases and their contacts. Research has shown that quarantine measures of infected individuals and their family members, combined with surveillance and standard testing procedures, are effective as control measures in the early stages of the pandemic (26). Individual mobility and contact (close proximity) data offer information about infected individuals, their locations and social network. Contact (close proximity) data can be collected through mobile apps $(24,27)$, interviews, or surveys $(28)$.

During the acceleration phase, when community transmission reaches exponential levels, the focus is on interventions for containment, which typically involve social contact and mobility restrictions. At this stage, aggregated mobile phone data are valuable to assess the efficacy of implemented policies through the monitoring of mobility between and within affected municipalities. Mobility information also contributes to the building of more accurate epidemiological models that can explain and anticipate the spread of the disease, as shown for H1N1 flu outbreaks (29). These models, in turn, can inform the mobilization of resources (e.g., respirators and intensive care units).

Finally, during the deceleration and preparation phases, as the peak of infections is reached, restrictions will likely be lifted (30). Continued situational monitoring will be important as the COVID-19 pandemic is expected to come in waves $(4,31)$. Near real-time data on mobility and hotspots will be important to understand how lifting and re-establishing various measures translate into behavior, especially to find the optimal combination of measures at the right time (e.g., general mobility restrictions, school closures, banning of large gatherings), and to balance these restrictions with aspects of economic vitality. After the pandemic has subsided, mobile data will be helpful for post-hoc analysis of the impact of different interventions on the progression of the disease and cost-benefit analysis of mobility restrictions. During this phase, digital contact tracing technologies might be deployed, such as the Korean smartphone app Corona $100 \mathrm{~m}$ (32) and the Singaporean smartphone app TraceTogether (33), that aim at minimizing the spread of a disease as mobility restrictions are lifted. Along this line, researchers at the Massachusetts Institute of Technology and collaborators are working on Private Kit: Safe Paths (25), an open-source and privacy-first contact-tracing technology that provides individuals with information on their proximity with diagnosed COVID-19 carriers, using GPS and Bluetooth data. Similarly, several European universities, research centers and companies have joined forces around PEPP-PT (Pan-European Privacy Preserving Proximity Tracing (34), a collaboration on privacy-preserving, GDPR-compliant contact tracing. Along this effort, a consortium of research institutions, led by the École Polytechnique Fédérale de Lausanne (EPFL), has developed an open Decentralized Privacy-Preserving Proximity Tracing (DP3T) protocol and implementation using Bluetooth Low Energy functionality on smartphones, ensuring that personal data and computation stays entirely on an individuals' phones (35). Recently, Apple and Google have released a joint announcement (36) describing their system to support Bluetooth based privacy-preserving proximity tracing across iOS and Android smartphones. Interestingly, as a part of the European Commission recommendation of a coordinated approach to support the gradual lifting of lockdown measures (37), European Union Members States, supported by the Commission, have developed a toolbox for the development and usage of contact tracing apps, fully compliant with EU rules (38).

\section{Specific metrics for data-supported decisions}

Researchers and practitioners have developed a variety of aggregated metrics using mobile phone data that can help fill gaps in information needed to respond to COVID-19 and address uncertainties regarding mobility and behaviors.

Origin-Destination (OD) matrices are especially useful in 
the first epidemiological phases, where the focus is to assess the mobility of the population. The number of people moving between two different areas daily can be computed from the mobile network data, and it can be considered a proxy of human mobility. The geographic areas of interest might be zip codes, municipalities, provinces or even regions. These mobility flows are compared to those during a reference period in order to assess the reduction in mobility due to non-pharmaceutical interventions. In particular, they are useful to monitor the impact of different social and mobility contention measures and to identify regions where the measures might not be effective or followed by the population. Moreover, these flows can inform spatially explicit disease transmission models to evaluate the potential benefit of such reductions.

Dwell estimations and hotspots are estimates of particularly high concentration of people in an area, which can be favorable to the transmission of the virus. These metrics are typically constructed within a municipality by dividing the city into grids or neighborhoods (39). The estimated number of people in each geographical unit can be computed with different time granularities (e.g., $15 \mathrm{~min}, 60 \mathrm{~min}, 24 \mathrm{~h}$ ).

Contact matrices estimate the number and intensity of the face-to-face interactions people have in a day. They are typically computed by age-groups. These matrices have been shown to be extremely useful to assess and determine the decrease of the reproduction number of the virus (6). However, it is still challenging to estimate face-to-face interactions from co-location and mobility data (40). Contact tracing apps can then be used to identify close contacts of those infected with the virus.

Amount of time spent at home, at work or other locations are estimates of the individual percentage of time spent at home/work/other locations (e.g., public parks, malls, shops), which can be useful to assess the local compliance with countermeasures adopted by governments. The home and work locations need to be computed in a period of time prior to the deployment of mobility restrictions measures. The percentage of time spent in each location needs to be computed for people who do not move during this time. Variations of the time spent on different locations is generally computed on an individual basis, and then spatially aggregated at a zip code, municipality, city or region level.

Although there is still little information about the age-specific susceptibility to COVID-19 infection, it is clear that age is an important risk factor for COVID-19 severity. We highlight, therefore, the importance of estimating the metrics mentioned above by age groups (6). Figure 2 shows an example of such metrics.

\section{Why is the use of mobile phone data not widespread, or a standard, in tackling epidemics?}

The use of mobile phone data for tackling the COVID-19 pandemic has gained attention but remains relatively scarce. Although local alliances have been formed, internationally concerted action is missing, both in terms of coordination and information exchange (22). In part, this is the result of a failure to institutionalize past experiences. During the 20142016 Ebola virus outbreak, several pilot or one-off activities were initiated. However, there was no transition to "business as usual" in terms of standardized procedures to leverage mobile phone data or establish mechanisms for "data readiness" in the country contexts $(41,42)$. Technology has evolved with various platforms offering enhanced, and secured access and analysis of mobile data, including for humanitarian and development use cases (e.g., OPAL [(43)], Flowkit [(44)]). Furthermore, high-level meetings have been held (e.g., the European Commission's B2G Data Sharing high-level expert group), data analysis and sharing initiatives have shown promising results, yet the use of metrics and insights derived from mobile phone data by governments and local authorities is still minimal today (43). Several factors likely explain this 'implementation' gap.

First, governments and public authorities frequently are unaware and/or lack a "digital mindset" and capacity needed for both for processing information that often is complex and requires multidisciplinary expertise (e.g., mixing location and health data, specialized modeling), as well as for establishing the necessary interdisciplinary teams and collaborations. Many government units are understaffed and sometimes also lack technological equipment. During the COVID-19 pandemic, most authorities are overwhelmed by the multiplicity and simultaneity of requests; as they have never been confronted with such a crisis, there are few predefined procedures and guides, so targeted and preventive action is quickly abandoned for mass actions. These problems are exacerbated at local levels of governments (e.g., towns, counties), which are precisely the authorities doing the front-line work in most situations. Additionally, many public authorities and decision-makers are not aware of the value that mobile phone data would provide for decision-making and are often used to make decisions without knowing the full facts and under conditions of uncertainty.

Second, despite significant efforts, access to data remains a challenge. Most companies, including mobile network operators, tend to be very reluctant to make data available-even aggregated and anonymized-to researchers and/or governments. Apart from data protection issues, such data are also seen and used as commercial assets, thus limiting the potential use for humanitarian goals if there are no sustainable models to support operational systems. One should also be aware that not all mobile network operators in the world are equal in terms of data maturity. Some are actively sharing data as a business, while others have hardly started to collect 
and use data.

Third, the use of mobile phone data raises legitimate public concerns about privacy, data protection and civil liberties. Governments in China, South Korea, Israel and elsewhere have openly accessed and used personal smartphone app data for tracking individual movements and for notifying individuals. However, in other regions, such as in Europe, both national and regional legal regulations limit such use (especially the European Union law on data protection and privacy known as the General Data Protection Regulation-GDPR). Furthermore, around the world, public opinion surveys, social media and a broad range of civil society actors including consumer groups and human rights organizations have raised legitimate concerns around the ethics, potential loss of privacy and long-term impact on civil liberties resulting from the use of individual mobile data to monitor COVID-19. Control of the pandemic requires control of people - including their mobility and other behaviors. A key concern is that the pandemic is used to create and legitimize surveillance tools used by government and technology companies that are likely to persist beyond the emergency. Such tools and enhanced access to data may be used for purposes such as law enforcement by the government or hyper-targeting by the private sector. Such an increase in government and industry power and the absence of checks and balance is harmful in any democratic state. The consequences may be even more devastating in less democratic states that routinely target and oppress minorities, vulnerable groups, and other populations of concern.

Fourth, researchers and technologists frequently fail to articulate their findings in clear, actionable terms that respond to practical political and technical questions. Researchers and domain experts tend to define the scope and direction of analytical problems from their perspective and not necessarily from the perspective of governments' needs. Critical decisions have to be taken, while key results are often published in scientific journals and in jargon that are not easily accessible to outsiders, including government workers and policy makers.

Finally, there is little political will and resourced invested to support preparedness for immediate and rapid action. On country levels, there are too few latent/standing mixed teams, composed of a) representatives of governments and public authorities, b) mobile network operators and technology companies, c) different topic experts (virologists, epidemiologists, data analysts); and there are no procedures and protocols predefined.

None of these challenges are insurmountable, but they require a clear call for action.

\section{A call to action to fight COVID-19}

To effectively build the best, most up to date, relevant, and actionable knowledge, we call on governments, mobile network operators and technology companies (e.g., Google, Facebook, Apple), and researchers to form mixed teams. Governments should be aware of the value of information and knowledge that can be derived from mobile phone data analysis, especially for monitoring the necessary measures to contain the pandemic. They should enable and leverage the fair and responsible provision/use of aggregated and anonymized data for this purpose. Mobile network operators and technology companies with widespread adoption of their products (e.g., Facebook, Google, Apple) should take their social responsibility and the vital role that they can play in tackling the pandemic. They should reach out to governments and the research community. Researchers and domain experts (e.g., virologists, epidemiologists, demographers, data scientists, computer scientists and computational social scientists) should acknowledge the value of interdisciplinary teams and context specificities and sensitivities. Impact would be maximized if governments and public authorities are included early on and throughout their efforts to identify the most relevant questions and knowledge needs. Creating multi-disciplinary inter-institutional teams is of paramount importance, as recently shown successfully in Belgium and the Valencian region of Spain (45). Four key principles should guide the implementation of such mixed teams in order to improve their effectiveness, namely: i) the early inclusion of governments, ii) the liaising with data protection authorities early on, iii) international exchange, and iv) preparation for all stages of the pandemic.

Relevant government and public authorities should be involved early, and researchers need to build upon their knowledge systems and need for information. One key challenge is to make insights actionable-how can findings such as propagation maps finally be utilized (e.g., for setting quarantine zones, informing local governments, targeting communication). At the same time, expectations must be realistic: decisions on measures should be based on facts, but are in the end, political decisions. Many insights derived from mobile phone data analytics do not have practical implications-such analysis and the related data collection should be discouraged until proven necessary.

We also suggest such efforts be transparent and involve data protection authorities and civil liberties advocates early on, and have quick iteration cycles with them. For example, policy makers should consider the creation of an ethics and privacy advisory committee to oversee and provide feedback on projects. This ensures that privacy is maintained and raises potential user acceptance. Aggregated mobile phone data can be used in line even with the strict European regulations (GDPR). Earlier initiatives have established principles and methods for sharing data or indicators without endangering personal information and build privacy-preserving 
solutions that use only incentives to manage behavior (4648). The early inclusion of the data protection authority in Belgium has led to the publishing of a statement by the European Data Protection Board on how to process mobile phone data in the fight against COVID-19 (49). Even while acknowledging the value of mobile phone data, the urgency of the situation should not lead to losses of data privacy and other civil liberties that might become permanent after the pandemic. In this regard, the donation of data for good, and the direct and limited (in time and scope) sharing of aggregated data by mobile network operators with (democratic) governments and researchers seems to be less problematic than the use of individual location data commercially acquired, brought together and analyzed by commercial enterprises. More generally, any emergency data system set to monitor COVID-19 and beyond must follow a balanced and well-articulated set of data policies and guidelines and is subjected to risk assessments.

Specifically, any efforts should meet clear tests on the proportionate, legal, accountable, necessary and ethical use of mobile phone data in the circumstances of the pandemic and seek to minimize the amount of information gathered to what is necessary to accomplish the objective concerned. These are not new criteria; they are well inscribed into international human rights standards and law concerning, for example, the use of force. Certainly, the use of mobile phone data does not equate to the use of force, but in the wrong hands, it can have similarly devastating effects and lead to significantly curtail civil liberties. Considering the broad absence of legal frameworks and historical mishandling of data by technology companies, there is an urgent need for responsible global leadership and governance to guide efforts to use technology in times of emergency.

We further see a clear need for more international exchange, with other domain experts, but also with other initiatives and groups; findings must be shared quickly-there will be time for peer-reviewed publications later. Especially in countries with weaker health (and often also economic) systems, the targeting and effectiveness of non-pharmaceutical interventions might make a big difference. This also implies the translation of important findings from English to other relevant languages.

For later stages of the pandemic, and for the future, stakeholders should aim for a minimum level of "preparedness" for immediate and rapid action. On country and/or region levels, there will be a need of "standing" mixed teams; up-todate technology, basic agreements and legal prescriptions; and data access, procedures and protocols predefined (also for "appropriate anonymization and aggregation protocols" (46)). A long-time collaboration between infectious disease modelers, epidemiologists, and researchers of mobile network operator labs in France helped jump-start a project on the COVID-19 pandemic, with the support of public health authorities (50).

Finally, in addition to (horizontal) international exchange, we also need international approaches that are coordinated by supranational bodies. National initiatives might help to a certain extent but will not be sufficient in the long run. A global pandemic necessitates globally or at least regionally coordinated work. Here, promising approaches are emerging: the EU Commission on 23 March 2020 called upon European mobile network operators to hand over anonymized and aggregated data to the Commission to track virus spread and determine priority areas for medical supplies (51), while other coordination initiatives are emerging in Africa, Latin America and the MENA-Region. It will be important for such initiatives to link up, share knowledge and collaborate. The Covid-19 pandemic will not be over soon, and it will not be the last pandemic we face. Privacy-aware and ethically acceptable solutions to use mobile phone data should be prepared and vetted in advance, and we must raise readiness on national and international levels, so we can act rapidly when the crisis hits.

\section{REFERENCES AND NOTES}

1. R. M. Anderson, H. Heesterbeek, D. Klinkenberg, T. D. Hollingsworth, How will country-based mitigation measures influence the course of the COVID-19 epidemic? Lancet 395, 931-934 (2020). doi:10.1016/S0140-6736(20)30567-5 Medline

2. M. Chinazzi, J. T. Davis, M. Ajelli, C. Gioannini, M. Litvinova, S. Merler, A. Pastore Y Piontti, K. Mu, L. Rossi, K. Sun, C. Viboud, X. Xiong, H. Yu, M. E. Halloran, I. M. Longini Jr., A. Vespignani, The effect of travel restrictions on the spread of the 2019 novel coronavirus (COVID-19) outbreak. Science 368, eaba9757 (2020). doi:10.1126/science.aba9757 Medline

3. L. D. Domenico, G. Pullano, P. Coletti, N. Hens, V. Colizza, Expected impact of school closure and telework to mitigate COVID-19 epidemic in France, 15.

4. N. Ferguson, D. Laydon, G. Nedjati Gilani, N. Imai, K. Ainslie, M. Baguelin, S. Bhatia, A. Boonyasiri, Z. Cucunuba Perez, G. Cuomo-Dannenburg, A. Dighe, I. Dorigatti, H. Fu, K. Gaythorpe, W. Green, A. Hamlet, W. Hinsley, L. Okell, S. Van Elsland, H. Thompson, R. Verity, E. Volz, H. Wang, Y. Wang, P. Walker, P. Winskill, C. Whittaker, C. Donnelly, S. Riley, A. Ghani, "Report 9: Impact of nonpharmaceutical interventions (NPIs) to reduce COVID19 mortality and healthcare demand" (Imperial College London, 2020), doi:10.25561/77482.

5. H. Tian, Y. Liu, Y. Li, C.-H. Wu, B. Chen, M. U. G. Kraemer, B. Li, J. Cai, B. Xu, Q. Yang, B. Wang, P. Yang, Y. Cui, Y. Song, P. Zheng, Q. Wang, O. N. Bjornstad, R. Yang, B. T. Grenfell, O. G. Pybus, C. Dye, An investigation of transmission control measures during the first 50 days of the COVID-19 epidemic in China. Science eabb6105 (2020). doi:10.1126/science.abb6105 Medline

6. J. Zhang, M. Litvinova, Y. Liang, Y. Wang, W. Wang, S. Zhao, Q. Wu, S. Merler, C. Viboud, A. Vespignani, M. Ajelli, H. Yu, "Age profile of susceptibility, mixing, and social distancing shape the dynamics of the novel coronavirus disease 2019 outbreak in China" (preprint. Epidemiology (2020). doi: 10.1101/2020.03.19.20039107

7. L. Bengtsson, J. Gaudart, X. Lu, S. Moore, E. Wetter, K. Sallah, S. Rebaudet, R. Piarroux, Using mobile phone data to predict the spatial spread of cholera. Sci. Rep. 5, 8923 (2015). doi:10.1038/srep08923 Medline

8. F. Finger, T. Genolet, L. Mari, G. C. de Magny, N. M. Manga, A. Rinaldo, E. Bertuzzo, Mobile phone data highlights the role of mass gatherings in the spreading of cholera outbreaks. Proc. Natl. Acad. Sci. U.S.A. 113, 6421-6426 (2016). doi:10.1073/pnas.1522305113 Medline

9. M. Tizzoni, P. Bajardi, A. Decuyper, G. Kon Kam King, C. M. Schneider, V. Blondel, Z. 
Smoreda, M. C. González, V. Colizza, On the use of human mobility proxies for modeling epidemics. PLOS Comput. Biol. 10, e1003716 (2014). doi:10.1371/iournal.pcbi.1003716 Medline

10. A. Wesolowski, N. Eagle, A. J. Tatem, D. L. Smith, A. M. Noor, R. W. Snow, C. O. Buckee, Quantifying the impact of human mobility on malaria. Science 338, 267270 (2012). doi:10.1126/science.1223467 Medline

11. A. Wesolowski, T. Qureshi, M. F. Boni, P. R. Sundsøy, M. A. Johansson, S. B. Rasheed, K. Engø-Monsen, C. O. Buckee, Impact of human mobility on the emergence of dengue epidemics in Pakistan. Proc. Natl. Acad. Sci. U.S.A. 112, 11887-11892 (2015). doi:10.1073/pnas.1504964112 Medline

12. Coronavirus, Lombardia monitora celle telefoniche. Adnkronos, (available at https://www.adnkronos.com/fatti/cronaca/2020/03/17/coronaviruslombardia-monitora-celle-telefoniche 7Ubs200MKGGSygaYEpLezO.html).

13. J. P. Colomé, Valencia prepara un proyecto pionero con datos de móviles para trazar el movimiento del coronavirus. EL PAÍS (2020), (available at https://elpais.com/tecnologia/2020-03-19/valencia-prepara-un-proyectopionero-con-datos-de-moviles-para-trazar-el-movimiento-delcoronavirus.html)

14. L. Ferres, R. Schifanella, N. Perra, S. Vilella, L. Bravo, D. Paolotti, G. Ruffo, M. Sacasa, "Measuring Levels of Activity in a Changing City" (2020), p. 11.

15. B. Klein, T. LaRock, S. McCabe, L. Torres, F. Privitera, B. Lake, M. U. G. Kraemer, D. Lazer, T. Eliassi-Rad, S. V. Scarpino, M. Chinazzi, A. Vespignani, "Assessing changes in commuting and individual mobility in major metropolitan areas in the United States during the COVID-19 outbreak," p. 29.

16. M. U. G. Kraemer, C.-H. Yang, B. Gutierrez, C.-H. Wu, B. Klein, D. M. Pigott, open COVID-19 data working group, L. du Plessis, N. R. Faria, R. Li, W. P. Hanage, J. S. Brownstein, M. Layan, A. Vespignani, H. Tian, C. Dye, S. Cauchemez, O. Pybus, S. V. Scarpino, "The effect of human mobility and control measures on the COVID19 epidemic in China" Science (2020). doi:10.1126/science.abb4218/

17. S. Lai, N. W. Ruktanonchai, L. Zhou, O. Prosper, W. Luo, J. R. Floyd, A. Wesolowski, M. Santillana, C. Zhang, X. Du, H. Yu, A. J. Tatem, "Effect of non-pharmaceutical interventions for containing the COVID-19 outbreak in China" (preprint, Infectious Diseases (except. HIV AIDS (Auckl.) (2020). 10.1101/2020.03.03.20029843

18. E. Pepe, P. Bajardi, L. Gauvin, F. Privitera, C. Cattuto, M. Tizzoni, COVID-19 outbreak response: first assessment of mobility changes in Italy following lockdown. COVID-19 Mobility Monitoring project (2020), (available at https://covid19mm.github.io/in-progress/2020/03/13/first-reportassessment.html).

19. M. Qian, A. Saunders, D. Pesch, S. Reece, W. D. Lee, X. Dong, R. Lambiotte, Oxford COVID-19 Impact Monitor, (available at https://www.oxford-covid-19.com/).

20. K. Lyons, Governments are using cellphone location data to manage the coronavirus. The Verge (2020), (available at https://www.theverge.com/2020/3/23/21190700/eu-mobile-carrierscustomer-data-coronavirus-south-korea-taiwan-privacy).

21. D. Martín-Calvo, A. Aleta, A. Pentland, Y. Moreno, "Effectiveness of social distancing strategies for protecting a community from a pandemic with a datadriven contact network based on census and real-world mobility data" (2020), p. 13 .

22. C. O. Buckee, S. Balsari, J. Chan, M. Crosas, F. Dominici, U. Gasser, Y. H. Grad, B. Grenfell, M. E. Halloran, M. U. G. Kraemer, M. Lipsitch, C. J. E. Metcalf, L. A. Meyers, T. A. Perkins, M. Santillana, S. V. Scarpino, C. Viboud, A. Wesolowski, A. Schroeder, Aggregated mobility data could help fight COVID-19. Science 368, 145-146 (2020). doi:10.1126/science.abb8021 Medline

23. The Unacast Social Distancing Scoreboard - Unacast, (available at https://www.unacast.com/post/the-unacast-social-distancing-scoreboard)

24. W. Dong, T. Guan, B. Lepri, C. Qiao, in Proceedings of the ACM on Interactive, Mobile, Wearable and Ubiquitous Technologies (2019; https://dl.acm.org/doi/10.1145/3328912), vol. 3, pp. 1-23.

25. R. Raskar, I. Schunemann, R. Barbar, K. Vilcans, J. Gray, P. Vepakomma, S. Kapa, A. Nuzzo, R. Gupta, A. Berke, D. Greenwood, C. Keegan, S. Kanaparti, R. Beaudry, D. Stansbury, B. B. Arcila, R. Kanaparti, V. Pamplona, F. M. Benedetti, A. Clough, R. Das, K. Jain, K. Louisy, G. Nadeau, V. Pamplona, S. Penrod, Y. Rajaee, A. Singh, G. Storm, J. Werner, "Apps Gone Rogue: Maintaining Personal Privacy in an Epidemic" (2020), (available at https://arxiv.org/abs/2003.08567).

26. J. R. Koo, A. R. Cook, M. Park, Y. Sun, H. Sun, J. T. Lim, C. Tam, B. L. Dickens,
Interventions to mitigate early spread of SARS-CoV-2 in Singapore: A modelling study. Lancet Infect. Dis. S1473-3099(20)30162-6 (2020). doi:10.1016/S14733099(20)30162-6 Medline

27. L. Ferretti, C. Wymant, M. Kendall, L. Zhao, A. Nurtay, L. Abeler-Dörner, M. Parker, D. Bonsall, C. Fraser, Quantifying SARS-CoV-2 transmission suggests epidemic control with digital contact tracing. Science eabb6936 (2020). doi:10.1126/science.abb6936 Medline

28. N. Oliver, X. Barber, K. Roomp, K. Roomp, "The Covid19 Impact Survey: Assessing the Pulse of the COVID-19 Pandemic in Spain via 24 questions," p. 11.https://arxiv.org/abs/2004.01014

29. D. Balcan, H. Hu, B. Goncalves, P. Bajardi, C. Poletto, J. J. Ramasco, D. Paolotti, N. Perra, M. Tizzoni, W. Van den Broeck, V. Colizza, A. Vespignani, Seasonal transmission potential and activity peaks of the new influenza A(H1N1): A Monte Carlo likelihood analysis based on human mobility. BMC Med. 7, 45 (2009). doi:10.1186/1741-7015-7-45 Medline

30. K. Kupferschmidt, Ending coronavirus lockdowns will be a dangerous process of trial and error. Science | AAAS (2020), (available at https://www. sciencemag.org/news/2020/04/ending-coronavirus-lockdownswill-be-dangerous-process-trial-and-error).

31. S. M. Kissler, C. Tedijanto, E. Goldstein, Y. H. Grad, M. Lipsitch, Projecting the transmission dynamics of SARS-CoV-2 through the postpandemic period. Science , eabb5793 (2020). 10.1126/science.abb5793 Medline

32. A. Dudden, A. Marks, South Korea took rapid, intrusive measures against Covid-19 and they worked. The Guardian (2020), (available at https://www.theguardian.com/commentisfree/2020/mar/20/south-korearapid-intrusive-measures-covid-19).

33. A. Kharpal, Use of surveillance to fight coronavirus raises concerns about government power after pandemic ends. CNBC (2020), (available at https://www.cnbc.com/2020/03/27/coronavirus-surveillance-used-bygovernments-to-fight-pandemic-privacy-concerns.html).

34. Pan-European Privacy-Preserving Proximity Tracing, Pepp Pt, (available at https://www.pepp-pt.org)

35. C. Troncoso, M. Payer, M. Salathé, J. Larus, D. W. Lueks, T. Stadler, D. A. Pyrgelis, D. Antonioli, L. Barman, S. Chatel, K. Paterson, S. Čapkun, D. Basin, D. J. Beutel, D. Jackson, K. Leuven, B. Preneel, N. Smart, D. D. Singelee, D. A. Abidin, T. Delft, S. Guerses, C. Cremers, "Decentralized Privacy-Preserving Proximity Tracing," $p$. 33.

36. Apple and Google partner on COVID-19 contact tracing technology - Apple, (available at https://www.apple.com/newsroom/2020/04/apple-and-googlepartner-on-covid-19-contact-tracing-technology/).

37. European Commission, "Joint European Roadmap towards lifting COVID-19 containment measures" (2020), (available at https://ec.europa.eu/info/sites/info/files/communication a_european_roadmap_to_lifting_coronavirus_containment_measures_0.pdf).

38. eHealth Network, Mobile applications to support contact tracing in the EU's fight against COVID-19: Common EU Toolbox for Member States (2020), (available at https:/ec.europa.eu/health/sites/health/files/ehealth/docs/covid19 apps en.pdf)

39. T. Louail, M. Lenormand, O. G. Cantu Ros, M. Picornell, R. Herranz, E. FriasMartinez, J. J. Ramasco, M. Barthelemy, From mobile phone data to the spatial structure of cities. Sci. Rep. 4, 5276 (2014). doi:10.1038/srep05276 Medline

40. M. Génois, A. Barrat, Can co-location be used as a proxy for face-to-face contacts? EPJ Data Sci. 7, 11 (2018). doi:10.1140/epjds/s13688-018-0140-1

41. Waiting on hold. The Economist (2014), (available at https://www.economist.com/science-and-technology/2014/10/27/waiting-onhold).

42. S. M. McDonald, "Ebola: A Big Data Disaster Privacy, Property, and the Law of Disaster Experimentation" (The Centre for Internet and Society, Bengaluru and Delhi, India, 2016), (available at https://cis-india.org/papers/ebola-a-big-datadisaster).

43. A. Maxmen, Can tracking people through phone-call data improve lives? Nature 569, 614-617 (2019). doi:10.1038/d41586-019-01679-5 Medline

44. D. Power, M. Thom, J. Gray, M. Albert, S. Delaporte, T. Li, J. Harrison, J. Greenhalgh, N. Thorne, L. Bengtsson, "FlowKit: Unlocking the power of mobile data for humanitarian and development purposes" (Flowminder, 2019), (available 
content/uploads/2019/02/FlowKit_UnlockingthePowerofMobileData.pdf).

45. J. Delcker, POLITICO Al: Decoded: How Al is helping fight a pandemic - Europe's coronavirus app - Insights from Valencia. POLITICO (2020), (available at https://www.politico.eu/newsletter/ai-decoded/politico-ai-decoded-how-ai-ishelping-fight-a-pandemic-europes-coronavirus-app-insights-from-valencia/).

46. C. O. Buckee, M. I. E. Cardenas, J. Corpuz, A. Ghosh, F. Haque, J. Karim, A. S. Mahmud, R. J. Maude, K. Mensah, N. V. Motaze, M. Nabaggala, C. J. E. Metcalf, S. A. Mioramalala, F. Mubiru, C. M. Peak, S. Pramanik, J. M. Rakotondramanga, E. Remera, I. Sinha, S. Sovannaroth, A. J. Tatem, W. Zaw, Productive disruption: Opportunities and challenges for innovation in infectious disease surveillance. BMJ Glob. Health 3, e000538 (2018). doi:10.1136/bmigh-2017-000538 Medline

47. Y.-A. de Montjoye, S. Gambs, V. Blondel, G. Canright, N. de Cordes, S. Deletaille, K. Engø-Monsen, M. Garcia-Herranz, J. Kendall, C. Kerry, G. Krings, E. Letouzé, M. Luengo-Oroz, N. Oliver, L. Rocher, A. Rutherford, Z. Smoreda, J. Steele, E. Wetter, A. S. Pentland, L. Bengtsson, On the privacy-conscientious use of mobile phone data. Sci. Data 5, 1-6 (2018). doi:10.1038/sdata.2018.286

48. E. Letouzé, N. Oliver, "Sharing is Caring Four Key Requirements for Sustainable Private Data Sharing and Use for Public Good" (Data-pop alliance and Vodafone Institute for Society and Communications, London, 2019), (available at https://datapopalliance.org/paper-sharing-is-caring-four-key-requirementsfor-sustainable-private-data-sharing-and-use-for-public-good/).

49. European Data Protection Board, "Statement on the processing of personal data in the context of the COVID-19 outbreak" (2020), (available at https://edpb.europa.eu/sites/edpb/files/files/file1/edpb statement 2020 pr ocessingpersonaldataandcovid-19_en.pdf).

50. Pourquoi les données téléphoniques aident à comprendre la pandémie de Covid19. Le Monde.fr (2020), (available at https://www.lemonde.fr/pixels/article/2020/03/27/pourquoi-les-donneestelephoniques-aident-a-comprendre-la-pandemie-de-covid196034708 4408996.html).

51. Commission tells carriers to hand over mobile data in coronavirus fight. POLITICO (2020), (available at https://www.politico.eu/article/european-commissionmobile-phone-data-thierry-breton-coronavirus-covid19/).

52. N. Qualls, A. Levitt, N. Kanade, N. Wright-Jegede, S. Dopson, M. Biggerstaff, C. Reed, A. Uzicanin; CDC Community Mitigation Guidelines Work Group, Community Mitigation Guidelines to Prevent Pandemic Influenza - United States, 2017. MMWR Recomm. Rep. 66, 1-34 (2017). doi:10.15585/mmwr.rr6601al Medline

Published First Release 27 April 2020

10.1126/sciadv.abc0764 


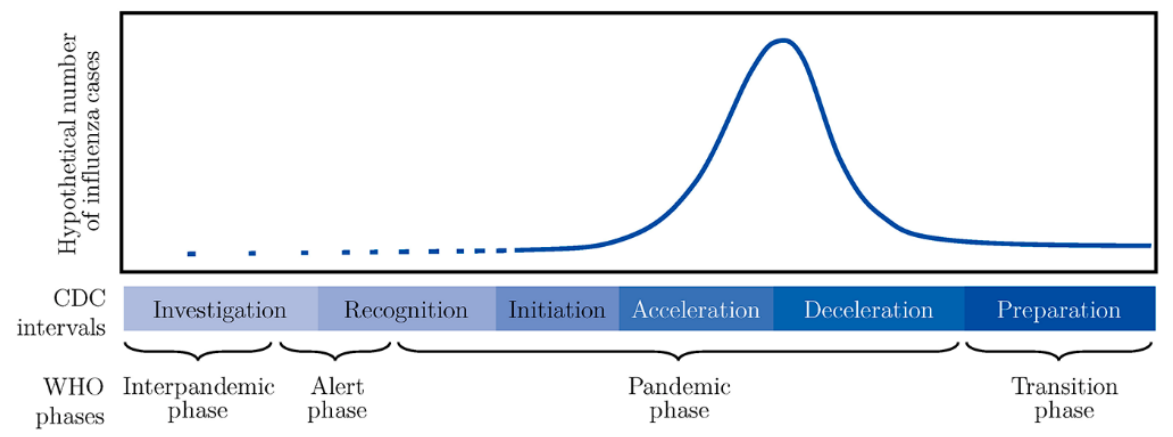

Fig. 1. Pandemic intervals as defined by the US Center for Disease Control and the WHO [based on (52)]. 


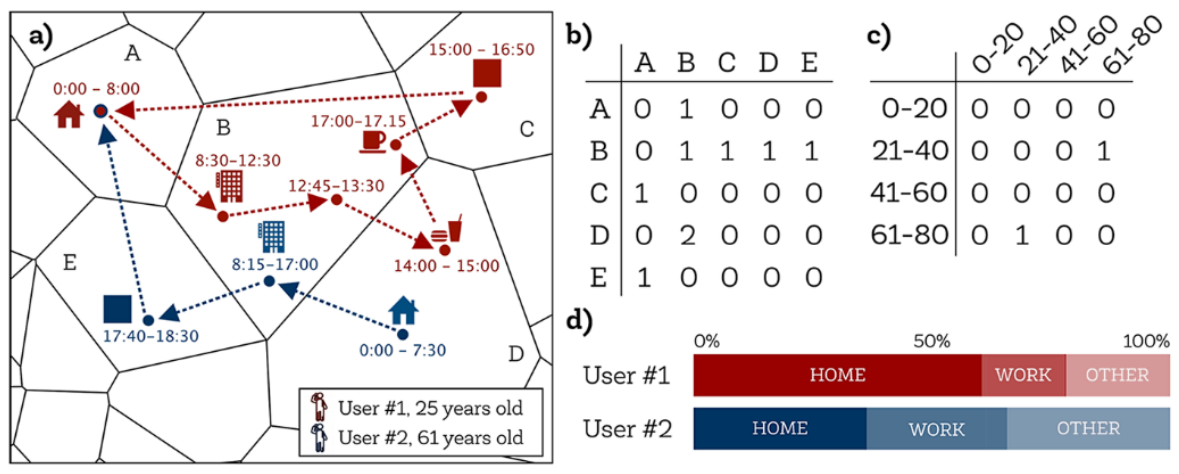

Fig. 2. Extraction of aggregated metrics from mobile phone data. a) Raw data representing one-day mobility of two users. In this example, the area $B$ is a hotspot, as it shows a high concentration of people. b) OriginDestination matrix of five different areas, counting the number of trips from one area (rows) to another area (columns). c) Contact matrix counting the number of potential face-to-face interactions between age groups. d) Percent of time spent at home, work and other locations. 
Table 1: Examples of questions by areas of enquiry

Situational awareness
- What are the most common mobility flows within and between COVID-affected cities and regions?

- Which areas are spreading the epidemics acting as origin nodes in a mobility network, and thus could be placed under mobility restrictions? - Are people continuing to travel or congregate after social distancing and travel restrictions were put into place?

- Are there hotspots at higher risk of contamination (due to a higher level of mobility, higher concentration of population)?

- What are the key entry points, locations and movements of roamers/tourists?

Predictive analysis

- How are certain human mobility patterns likely to affect the spread of the coronavirus? And what is the likely spread of COVID-19, based on existing disease models and up-to-date mobility data?

- What are the likely effects of mobility restrictions on children's education outcomes? - What are likely to be the economic consequences of restricted mobility for businesses?
Cause-and-effect

- What are variables that determine the success of social distancing approaches?

- How do local mobility patterns impact the burden on the medical system?

- Are business' social distancing recommendations resulting in more workers working from home?

- In what sectors are people working most from home?

- What are the social and economic consequences of movement restriction measures?

Impact

- How have travel restrictions impacted human mobility behavior and likely disease transmission?

- What is the potential of various restriction measures to avert infection cases and save lives?

- What is the effect of mandatory social distancing measures, including closure of schools?

- How has the dissemination of public safety information and voluntary guidance impacted human mobility behavior and disease spread? 